\section{Tendências de mortalidade por câncer de boca e orofaringe no Município de São Paulo, Brasil, 1980/2002}

\author{
Trends in oral cancer mortality in the city \\ of São Paulo, Brazil, 1980-2002
}

Maria Gabriela Haye Biazevic 1,2 Roberto Augusto Castellanos 2 José Leopoldo Ferreira Antunes 3 Edgard Michel-Crosato ${ }^{1}$

\author{
1 Área das Ciências \\ Biológicas e da Saúde, \\ Universidade do Oeste \\ de Santa Catarina, \\ Joaçaba, Brasil. \\ 2 Faculdade de Saúde \\ Pública, Universidade de \\ São Paulo, São Paulo, Brasil. \\ 3 Faculdade de Odontologia \\ Universidade de São Paulo, \\ São Paulo, Brasil. \\ Correspondência \\ M. G. H. Biazevic \\ Área das Ciências Biológicas \\ e da Saúde, Universidade \\ do Oeste de Santa Catarina. \\ Rua Getúlio Vargas 2125, \\ Joaçaba, SC \\ 89600-000, Brasil. \\ gabriela@unoescjba.edu.br
}

\begin{abstract}
The current study assessed trends in oral cancer mortality in the city of São Paulo, Brazil, from 1980 to 2002. The official mortality information system supplied data on deaths whose underlying cause was classified as oral cancer, stratified by sex, age, and anatomic site. Death rates were estimated and adjusted by the direct method, using population data supplied by national censuses from 1980, 1991, and 2000 and a population count performed in 1996. There was an upward trend in overall cancer mortality, at a yearly rate of $0.72 \%$. Accounting for more than one third of these deaths, tongue cancer was the main mortality category. Labial, gengival, and retromolar cancer showed a downward trend, while oropharyngeal cancer and cancer in unspecified parts of the mouth and oropharynx showed increasing mortality. Monitoring the magnitude and trends in cancer mortality can assist the planning of health initiatives aimed at reducing the disease burden from oral cancer in Brazil.
\end{abstract}

Mouth Neoplasms; Mortality; Statistical Models

\section{Introdução}

Segundo a Organização Mundial da Saúde (OMS), o câncer de boca e orofaringe são considerados os neoplasmas mais freqüentes de cabeça e pescoço, com cerca de 390 mil novos casos por ano ${ }^{1}$. A designação comum de "câncer de boca e orofaringe" refere-se a uma categoria abrangente de localização de neoplasias com diferentes etiologias e perfis histológicos, embora majoritariamente se refira ao carcinoma epidermóide. Sua etiologia é multifatorial, integrando fatores endógenos, como a predisposição genética e fatores exógenos ambientais e comportamentais, de cuja integração pode resultar a manifestação do agravo. A doença afeta majoritariamente as pessoas com mais de 45 anos de idade e, internacionalmente, há muita variação inter e intra-regional de incidência 2 .

Hamada et al. 3 afirmaram que o álcool e o tabaco seriam responsáveis por níveis de incidência de câncer bucal no Brasil comparáveis aos da Índia, país em que o risco mais elevado tem sido apontado como associado ao hábito de mascar tabaco. Também Franceschi et al. 4 classificaram informações sobre a incidência de câncer de boca e de orofaringe no Brasil dentre as mais elevadas em nível mundial; e Wünsch Filho 5 relatou taxas de incidência na cidade de São Paulo, Brasil, como sendo ainda mais elevadas que em outras cidades brasileiras. De 1997 a 1999, o número de novos casos da doença (re- 
ferindo-se aos códigos C00 a C10 da Classificação Internacional de Doenças, $10 \underline{a}$ revisão ${ }^{6}$ ) correspondeu a $5,7 \%$ e $2,26 \%$ de todas as neoplasias malignas (exceto tumores de pele), respectivamente para homens e mulheres na cidade de São Paulo ?.

Além de refletir o impacto diferencial dos fatores de risco, o estudo de mortalidade por neoplasias é influenciado por variações de quantidade e qualidade dos serviços de saúde. Níveis mais ou menos elevados de provisão, acesso e efetividade dos serviços de saúde podem propiciar condições mais favoráveis para a prevenção, para o diagnóstico precoce e redução de incapacidades, para a implementação dos recursos terapêuticos e um melhor prognóstico para os pacientes afetados. No entanto, o monitoramento destas dimensões, assim como o estudo da distribuição diferencial dos fatores de risco, é difícil de ser realizado em larga escala e período extenso.

Nesse sentido, o estudo de séries temporais da mortalidade por câncer de boca e orofaringe foi a estratégia empregada para caracterizar variações na carga de doença e fornecer indicações sobre possíveis fatores associados a estas variações. O presente estudo teve como objetivos descrever e explorar analiticamente a magnitude e as tendências da mortalidade por câncer de boca e orofaringe no Município de São Paulo no período de 1980 a 2002, com discriminação para sexo, diferentes grupos etários e categorias de localização anatômica.

\section{Métodos}

Foram coletados dados sobre os óbitos de pessoas residentes no Município de São Paulo, ocorridos entre 1980 e 2002, cuja causa básica foi identificada pelo Sistema de Informações sobre Mortalidade (SIM) como sendo câncer de boca e orofaringe. Os diferentes sítios de localização anatômica das neoplasias foram identificados pela Classificação Internacional de Doenças, segundo os códigos empregados pela 9a (CID-9 8) e 10a (CID-10 6) revisões. A mudança do sistema classificatório implicou apenas alterações dos códigos empregados: câncer de lábio (140 na CID-9 e C00 na CID-10), língua (141 e C01, C02), gengiva (143 e C03), pálato e úvula (145.2 a 145.5 e C05), mucosa oral (145.0 e C06.0), vestíbulo da boca (145.1 e C06.1), área retromolar (145.6 e C06.2), glândulas salivares maiores (142 e C07, C08), amígdala (146.0 a 146.2 e C09), orofaringe (146.3 a 146.9 e C10), outras partes e partes não especificadas da boca (145.8, 145.9, 149.8, 149.9 e C06.8, C06.9, C14.8).
Os dados sobre óbitos na cidade de São Paulo foram levantados junto à Fundação Sistema Estadual de Análise de Dados (SEADE), gestora do sistema oficial de informação de mortalidade no Estado de São Paulo, o qual foi recentemente avaliado como uma fonte confiável de informações 9 . A contagem da população 10 realizada em 1996 e os censos demográficos realizados em 1980 11, 199112 e 2000 13, sob a coordenação do Instituto Brasileiro de Geografia e Estatística (IBGE), forneceram informações sobre o número de moradores na cidade de São Paulo, discriminados por sexo e grupo etário. Para os anos intercensitários, a população foi estimada pelo método da progressão geométrica 14 , com ajuste anual levando em consideração as datas base estipuladas para as medidas primárias de população e o meio de cada período para o qual os coeficientes foram calculados. Todos os coeficientes descritos e analisados foram calculados usando 100 mil habitantes como base de população.

Para evitar que modificações recentes no perfil etário da população residente na cidade 15,16 interferissem na estimação de tendências e na comparação de dados colhidos em diferentes períodos, foram empregadas duas estratégias: o cálculo de coeficientes específicos por dois grandes grupos etários (60 anos ou mais e 40 a 59 anos) e o ajuste dos coeficientes por sexo e grupo etário (de cinco em cinco anos até 70 anos ou mais) segundo o método direto 14 .

Visando expressar as estimativas do risco de morte por câncer de boca e orofaringe segundo medidas cuja ordem de grandeza fosse comparável à mortalidade recente na cidade, $o$ ajuste dos coeficientes tomou como padrão a distribuição etária e por sexo da população estimada para a cidade como um todo no período mais recente (2002). Esta opção permitiu atribuir validade interna às comparações no tempo efetuadas no âmbito do presente estudo, mas não autoriza comparações diretas com coeficientes não-ajustados calculados para outras populações, outros períodos ou mesmo para coeficientes ajustados usando outros padrões de distribuição etária.

Para cada localização anatômica de câncer de boca e orofaringe, foram delineadas séries temporais para os coeficientes ajustados de mortalidade e específicos por grupo etário para ambos os sexos. A apreciação visual de tendências em representações gráficas de séries temporais é, muitas vezes, prejudicada pela rugosidade do polígono de freqüências delineado. Esta rugosidade é causada pelo movimento de variação aleatória que, em adição à tendência, se faz presente na série temporal. Para evi- 
tar que esse efeito prejudicasse a aferição visual das tendências, os gráficos delineados empregaram o recurso de alisamento por médias móveis 17 de ordem 3. Como exceção, o gráfico representando as séries cuja magnitude de mortalidade foi menos elevada (lábios, gengiva e área retromolar) empregou alisamento por médias móveis de ordem 5 .

A estimação de tendências seguiu indicações metodológicas apresentadas por Antunes \& Waldman 18. O cálculo das taxas de crescimento ou declínio anual utilizou o procedimento de Prais-Winsten 17 para regressão linear generalizada, o qual permite efetuar correção de autocorrelação de primeira ordem na análise de séries de valores organizados no tempo. Para efetuar controle sobre a efetividade do uso desta opção metodológica, empregou-se o teste de Durbin-Watson 17, para dimensionar a existência de autocorrelação de primeira ordem da série temporal composta pelos coeficientes anuais, como para verificar se a correção efetuada era compatível com a hipótese de resíduos de regressão com distribuição aleatória.

Este procedimento possibilitou classificar os tipos de câncer de boca e orofaringe, interpretando como crescente, decrescente ou estacionária a tendência recente de mortalidade, além de quantificar a taxa de incremento anual da medida. Para essa finalidade, avaliou-se a tendência global do período estudado, em bus- ca de indicações de fatores estruturais que pudessem explicar a variação na mortalidade.

Para os procedimentos estatísticos foi utilizado o programa SPSS (SPSS Inc., Chicago, Estados Unidos). O estudo envolveu apenas a coleta e análise documental de dados secundários e de literatura, tendo sido aprovado pelo Comitê de Ética em Pesquisa da Faculdade de Saúde Pública, Universidade de São Paulo.

\section{Resultados}

Os coeficientes ajustados por sexo e faixa etária da mortalidade por câncer de boca e orofaringe no Município de São Paulo apresentaram tendência de incremento no período estudado. $\mathrm{O}$ câncer de língua foi a categoria com maior concentração de óbitos, correspondendo a pouco mais de um terço do total (Tabela 1). Câncer de orofaringe e câncer em outras partes e partes não especificadas também contabilizaram elevada participação percentual na mortalidade por câncer de boca e orofaringe. As localizações anatômicas que apresentaram menor proporção de óbitos foram mucosa oral e vestíbulo da boca.

A mortalidade global por câncer de boca e orofaringe foi em média 4,75 vezes mais elevada no sexo masculino que no feminino. O estudo de tendências indicou incremento da mortalidade global a uma taxa anual de $0,72 \%$, e di-

Tabela 1

Mortalidade por câncer de boca e orofaringe, segundo localização anatômica. Município de São Paulo, Brasil, 1980/2002. Freqüências absolutas e relativas de óbitos no período, e média anual dos coeficientes ajustados por sexo e grupo etário (por 100 mil habitantes).

\begin{tabular}{lccc}
\hline Localização anatômica & Número de óbitos & $\%$ & $\begin{array}{c}\text { Média dos } \\
\text { coeficientes anuais }\end{array}$ \\
\hline Lábios & 82 & 1,34 & 0,05 \\
Língua & 2.193 & 35,85 & 1,15 \\
Gengiva & 105 & 1,72 & 0,06 \\
Assoalho da boca & 360 & 5,89 & 0,19 \\
Palato & 358 & 5,85 & 0,19 \\
Mucosa oral* & 29 & 0,47 & 0,07 \\
Vestíbulo da boca* & 4 & 1,83 & 0,06 \\
Área retromolar & 112 & 6,28 & 0,21 \\
Glândulas salivares maiores & 384 & 8,24 & 0,26 \\
Amígdala & 504 & 19,65 & 0,60 \\
Orofaringe & 1.202 & 12,81 & 0,40 \\
Parte mal definida ou não especificada & 784 & 100,00 & 3,21 \\
Global & 6.117 & & \\
\hline
\end{tabular}

* O número de óbitos associados a vestíbulo da boca e mucosa oral foi muito pequeno para permitir a estimação de coeficientes anuais. 
ferentes indicações para o estudo específico por localização anatômica: declínio para lábios $(-8,3 \%)$, gengiva $(-5,46 \%)$ e área retromolar $(-3,75 \%)$; estacionária para língua, assoalho de boca, palato, glândulas salivares maiores, e amígdala; e de aumento para orofaringe $(+4,51 \%)$ e partes não especificadas da boca e orofaringe $(+4,44 \%)$ (Tabela 2). O reduzido número de óbitos por câncer de mucosa oral e vestíbulo da boca não permitiu a estimação de tendências para a mortalidade por esses tipos da doença.

A Tabela 3 sintetiza o estudo de tendências para os coeficientes de mortalidade específicos para os grupos etários de 40 a 59 anos e de 60 anos ou mais. De modo geral, as tendências identificadas para os coeficientes específicos por grupo etário foram consistentes com as tendências estimadas para os coeficientes ajustados por sexo e grupo etário. Apesar do crescimento identificado para a taxa global ajustada, a mortalidade por câncer de boca e orofaringe não foi estacionária apenas para o grupo de homens com idade mais elevada. Ainda a título de sublinhar indicações discrepantes, observou-se que apesar da tendência estacionária para câncer de assoalho da boca e amígdala, houve declínio significante da mortalidade nestas categorias, para o grupo etário de 40 a 59 anos.

A Figura 1 mostra as séries temporais da mortalidade por câncer de boca e orofaringe com discriminação por sexo e pelos dois grupos etários singularizados para análise. Foram destacadas para apresentação gráfica as três categorias com maior participação percentual na mortalidade por câncer de boca e orofaringe (de língua, Figura 2a; orofaringe, Figura 2b; e partes não especificadas, Figura 2c); e as três categorias com declínio de mortalidade no período (lábios, gengiva e área retromolar: Figura 3).

\section{Discussão}

O presente estudo apóia-se na premissa de que o reconhecimento de variações da carga de doença pode fornecer indicações importantes sobre fatores de modificação de risco. Esta finalidade, no entanto, pode ser dificultada pela variação no tempo da capacidade de elucidação diagnóstica e da qualidade do sistema de notificação dos óbitos. O sub-registro de mortes devidas ao câncer pode resultar da pouca exatidão na definição da causa de morte e taxas reduzidas de mortalidade pela doença podem estar associadas a uma proporção mais elevada de óbitos devidos a causas mal definidas ou não especificadas no conjunto de registro de mortes.

Não obstante, pondera-se que os dados coletados constituem a melhor informação sobre mortalidade por câncer na cidade, disponível

Tabela 2

Taxa de crescimento anual dos coeficientes ajustados de mortalidade por câncer de boca e orofaringe segundo localização anatômica e sexo. Município de São Paulo, Brasil, 1980/2002.

\begin{tabular}{lccc}
\hline Variáveis & $\begin{array}{c}\text { Taxa de crescimento } \\
\text { anual (\%) }\end{array}$ & IC95\% & Tendência \\
\hline $\begin{array}{l}\text { Localização anatômica } \\
\text { Lábios }\end{array}$ & $-8,30$ & $-9,53 ;-7,06$ & decrescente \\
Língua & $+0,12$ & $-0,40 ; 0,64$ & estacionária \\
Gengiva & $-5,46$ & $-8,95 ;-1,84$ & decrescente \\
Assoalho da boca & $-1,77$ & $-3,99 ; 0,50$ & estacionária \\
Palato & $+0,02$ & $-1,51 ; 1,56$ & estacionária \\
Área retromolar & $-3,75$ & $-6,51 ;-0,92$ & decrescente \\
Glândulas salivares & $-0,41$ & $-1,90 ; 1,10$ & estacionária \\
Amígdala & $-1,89$ & $-3,96 ; 0,22$ & estacionária \\
Orofaringe & $+4,51$ & 3,$09 ; 5,95$ & crescente \\
Parte mal definida ou não especificada & $+4,44$ & 3,$31 ; 5,58$ & crescente \\
Sexo & & & estacionária \\
Feminino & & $-0,02 ; 1,84$ & crescente
\end{tabular}


Tabela 3

Tendência de mortalidade por câncer de boca e orofaringe. Município de São Paulo, Brasil, 1980/2002.

Taxa de crescimento anual dos coeficientes específicos por grupo etário, sexo e localização anatômica.

\begin{tabular}{|c|c|c|c|c|}
\hline \multirow[t]{2}{*}{ Variáveis } & \multicolumn{2}{|c|}{40 a 59 anos } & \multicolumn{2}{|c|}{60 ou mais anos } \\
\hline & Crescimento anual (\%) & IC95\% & Crescimento anual (\%) & IC95\% \\
\hline \multicolumn{5}{|l|}{ Localização anatômica } \\
\hline Lábios & $-12,28$ & $-14,88 ;-9,60$ & $-7,80$ & $-9,40 ;-6,17$ \\
\hline Língua & 0,05 & $-068 ; 0,77$ & $-0,35$ & $-1,03 ; 0,34$ \\
\hline Gengiva & $-7,30$ & $-10,06 ;-4,45$ & $-5,00$ & $-8,64 ;-1,21$ \\
\hline Assoalho da boca & $-2,78$ & $-4,46 ;-3,49$ & $-1,56$ & $-4,94 ; 1,93$ \\
\hline Palato & $-1,60$ & $-3,49 ; 0,32$ & 2,31 & $-0,26 ; 4,94$ \\
\hline Área retromolar & $-3,32$ & $-5,53 ;-1,04$ & $-5,53$ & $-10,38 ; 0,41$ \\
\hline Glândulas salivares & $-2,05$ & $-5,15 ; 1,15$ & 0,42 & $-1,21 ; 2,09$ \\
\hline Amígdala & $-3,25$ & $-5,29 ;-1,17$ & 0,32 & $-2,25 ;-2,95$ \\
\hline Orofaringe & 4,85 & 3,$78 ; 5,92$ & 3,91 & 1,$68 ; 6,18$ \\
\hline $\begin{array}{l}\text { Parte mal definida ou } \\
\text { não especificada }\end{array}$ & 3,11 & 2,$12 ; 4,11$ & 5,28 & 3,$30 ; 7,29$ \\
\hline \multicolumn{5}{|l|}{ Sexo } \\
\hline Feminino & 1,05 & $-0,37 ; 2,49$ & 0,49 & $-0,30 ; 1,28$ \\
\hline Masculino & 0,43 & $-0,10 ; 0,95$ & 0,96 & 0,$35 ; 1,58$ \\
\hline Global & 0,32 & $-0,19 ; 0,84$ & 0,64 & 0,$06 ; 1,22$ \\
\hline
\end{tabular}

Figura 1

Mortalidade (por 100 mil habitantes) por câncer de boca e orofaringe. Município de São Paulo,

Brasil, 1980/2002. Coeficientes específicos por sexo e grupo etário.

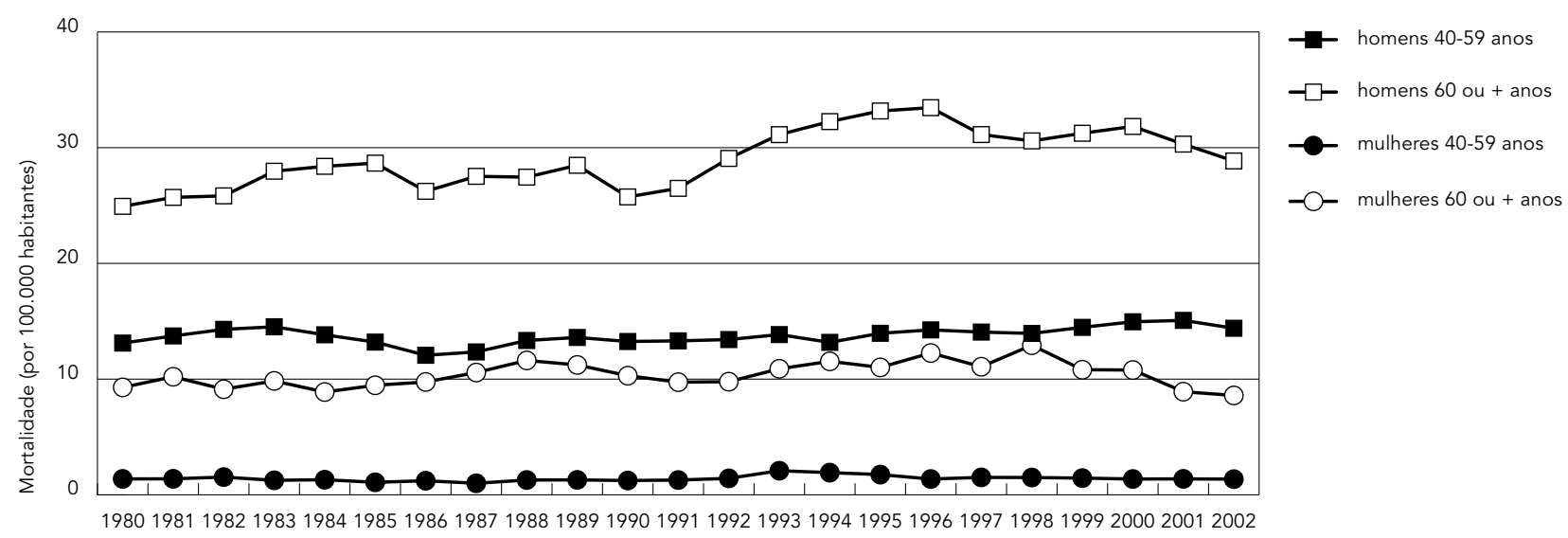




\section{Figura 2}

Mortalidade (por 100 mil habitantes) por câncer de língua, orofaringe e partes não especificadas da boca e orofaringe. Município de São Paulo, Brasil, 1980/2002.

Figura 2a) Língua

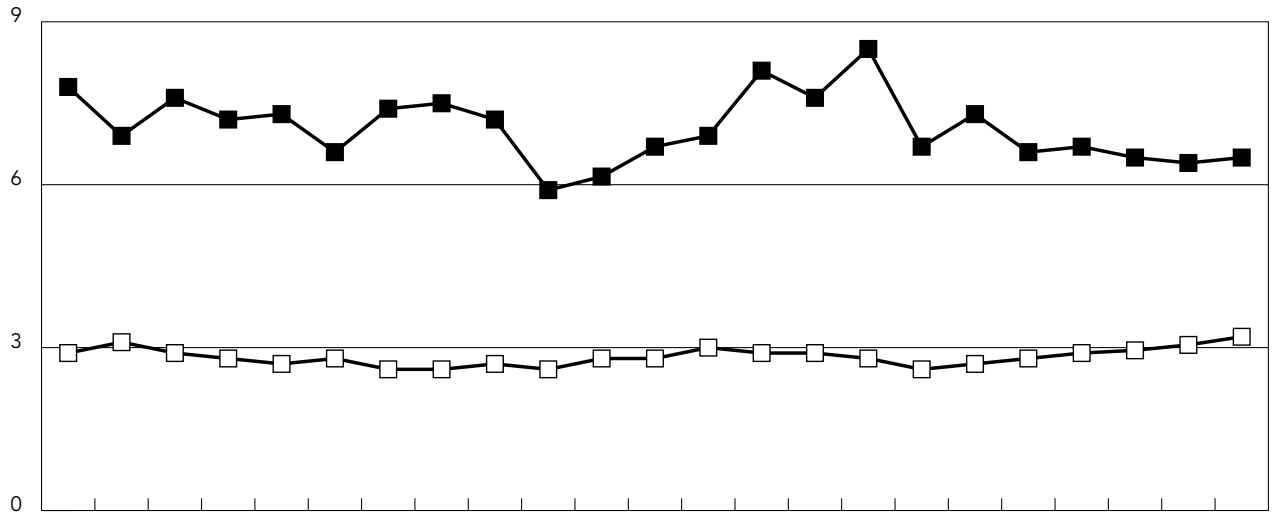

-60 anos ou +

$-\square-40-59$ anos

19801981198219831984198519861987198819891990199119921993199419951996199719981999200020012002

Figura 2b) Orofaringe

4,5

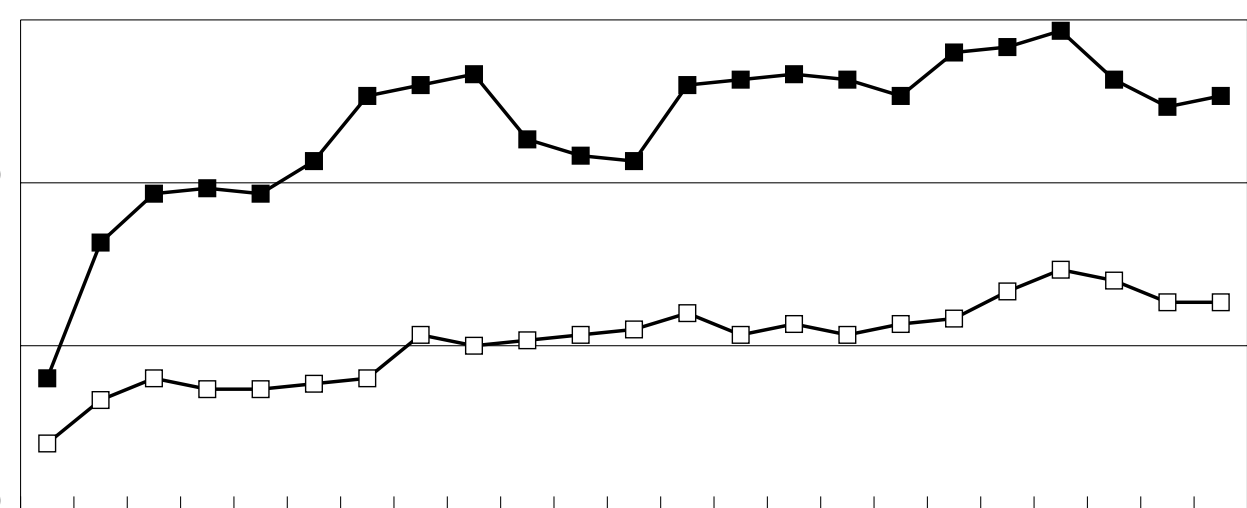

19801981198219831984198519861987198819891990199119921993199419951996199719981999200020012002

Figura 2c) Partes não especificadas

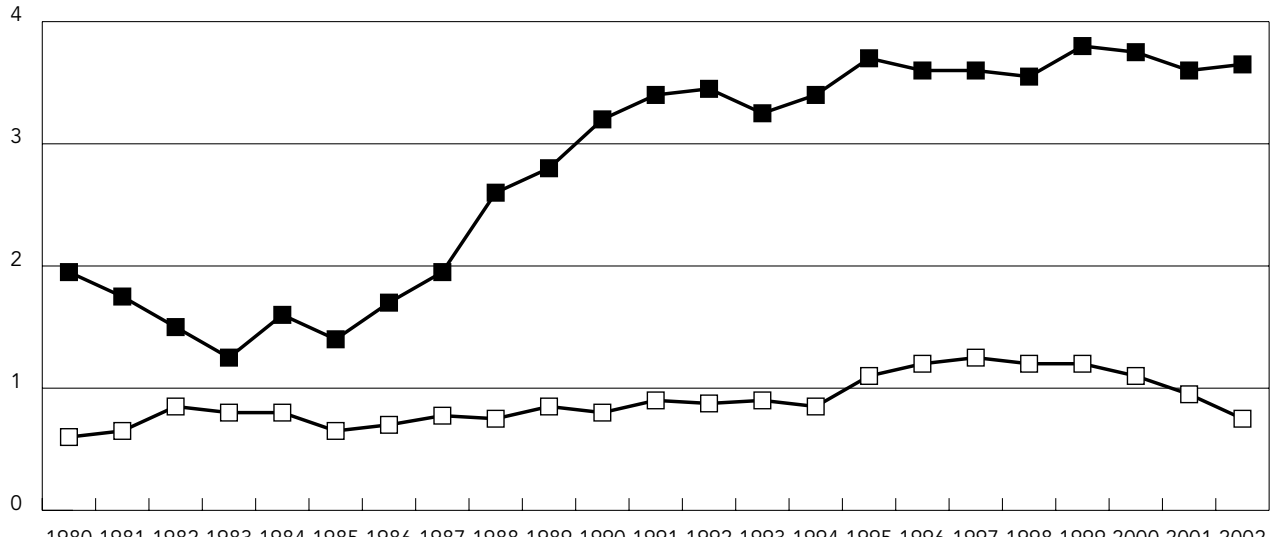

60 anos ou +

$-\square-40-59$ anos

19801981198219831984198519861987198819891990199119921993199419951996199719981999200020012002 
Mortalidade (por 100 mil habitantes) por câncer de lábios, gengiva e área retromolar. Município de São Paulo, Brasil, 1980/2002.

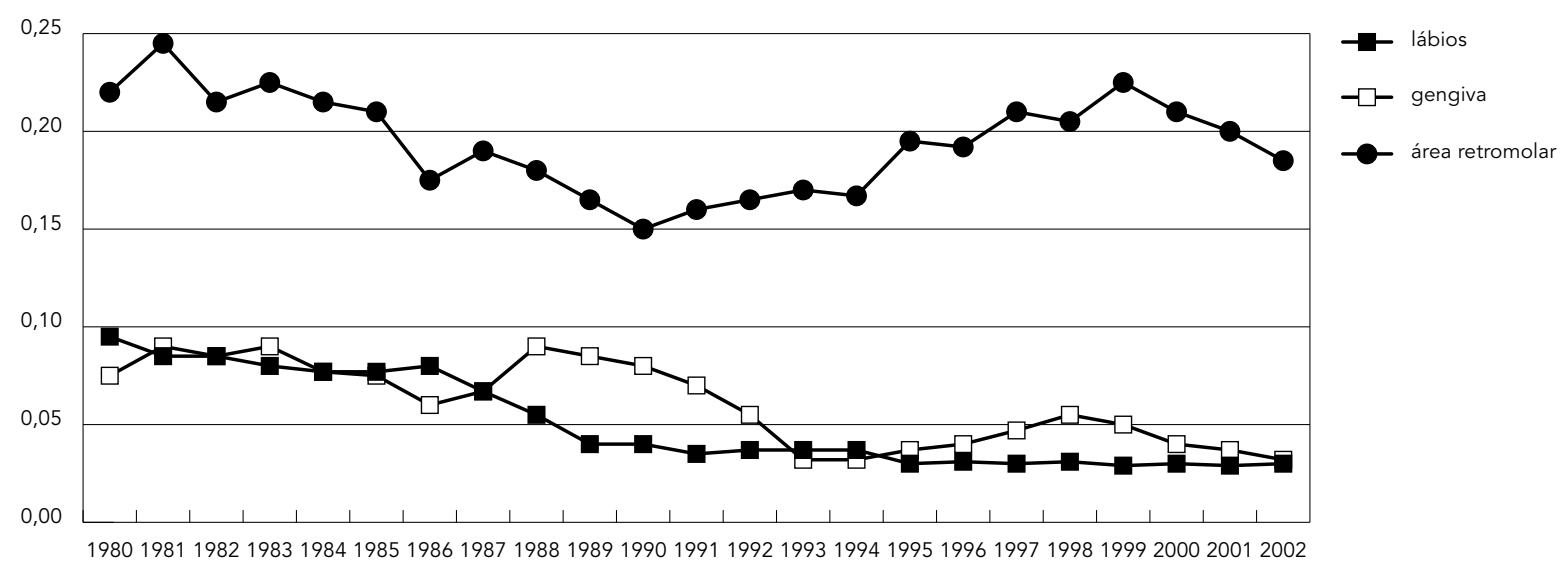

para o planejamento dos serviços de saúde. Reforçando esta convicção, estudos recentes de demografia avaliaram como precisas ou de boa qualidade tanto as informações de população como as de mortalidade no Estado de São Paulo e na Região Sudeste do Brasil durante o período analisado 19,20.

O estudo de tendências da mortalidade por câncer é complexo e deve considerar fatores de natureza diversa. Essas tendências decerto refletem níveis diferenciais de qualidade e acesso aos serviços de saúde, além da exposição a fatores de risco e proteção. Entretanto, esses fatores também sofrem modificação de magnitude e freqüência ao longo do tempo; e a implementação de suas conseqüências potencialmente benéficas ou deletérias depende de ação cumulativa. Como resultado, seus efeitos podem afetar distintos grupos de população com mais ou menos intensidade; e a avaliação de risco específico para diferentes cortes de nascimento possibilitaria uma consideração mais extensa sobre as tendências de mortalidade. Apesar dessa ponderação, o presente estudo optou por um esquema analítico mais simples e as séries temporais foram delineadas com base em recortes transversais organizados no tempo.

Foi constatado incremento de mortalidade por câncer de boca e orofaringe na cidade de 1980 a 2002 (Tabela 2). Esta observação demanda cuidadosa consideração por parte dos serviços de saúde, pois suscita apreensões de natureza diversa. Além de ter sido considerada ele- vada a magnitude desta mortalidade, em comparações envolvendo dados para o contexto nacional e internacional 21,22, é presumível que a tendência crescente dos coeficientes ajustados por idade esteja associada a crescimento ainda mais elevado da mortalidade bruta. Esta observação reflete a mudança de perfil etário da população, com a crescente participação percentual dos grupos de idade mais avançada, e indica crescimento da demanda por serviços de saúde associados ao câncer de boca e orofaringe.

Do ponto de vista dos principais fatores comportamentais reconhecidos como de risco para o agravo, o registro de mortalidade crescente para a doença na cidade indica o muito que ainda há a ser feito para o controle do tabagismo e do alcoolismo, e para a redução de seus efeitos deletérios sobre a saúde coletiva. É interessante notar que a tendência global de incremento concentrou-se sobre homens com 60 anos ou mais, grupo sobre o qual pesaria com mais intensidade o tempo acumulado de exposição a esses fatores.

Estima-se que um terço da população de adultos no Brasil seja fumante 23 . O consumo per capita anual de cigarros, calculado pelo quociente entre o total produzido, descontando a porção destinada à exportação e acrescendo a importação do produto, e a população com 15 ou mais anos de idade, foi avaliado como relativamente baixo na comparação com outros países, apesar de ter crescido na década de 8024 e de 90 25. Ademais, estima-se que esses dados 
estariam fortemente subestimados, em função de a entrada ilegal de cigarros fabricados no país para exportação ao Paraguai atingir de 31 a 37\% da comercialização do produto no Brasil 25.

No que diz respeito ao alcoolismo, sua participação no risco de neoplasias do trato aéreodigestivo superior foi descrita no Brasil não apenas como fator independente, mas como efeito de interação com o tabaco 26. Agravando o problema do álcool como fator de risco para câncer, estudos no país têm apontado maior prevalência de fumantes entre as pessoas submetidas ao consumo habitual de bebidas alcoólicas 27. Estas observações são compatíveis com a persistência de níveis mais elevados para homens da mortalidade por câncer de boca e orofaringe, uma vez que a prevalência de consumo de bebidas alcoólicas em níveis potencialmente danosos à saúde foi descrita como sendo cerca de cinco vezes mais elevada em homens que em mulheres na cidade de São Paulo 28.

A observação de elevada magnitude e tendência crescente para a mortalidade por câncer de localizações não especificadas da boca e orofaringe é sugestiva de retardo no diagnóstico e no estabelecimento de medidas terapêuticas, devendo, portanto, ser matéria de extensa consideração para os profissionais de saúde. Vários estudos partilham esta apreensão. Kowalski 29 afirmou que, embora passível de prevenção e detectável em estágios iniciais, o carcinoma bucal não tem recebido suficiente atenção por parte da população e dos profissionais de saúde. Vanderlei et al. 30 verificaram que muitos casos são diagnosticados em estágios avançados, apesar de serem visíveis e palpáveis com facilidade.

A indicação favorável de declínio da mortalidade por câncer de lábios, gengiva e área retromolar deve servir de incentivo para a implementação dos programas de câncer de boca e orofaringe na cidade. Como os tumores nesses sítios anatômicos são os mais facilmente identificáveis durante o exame bucal de rotina, este declínio poderia estar refletindo a expansão de cobertura dos atendimentos dentários no período de estudo, no qual se assistiu a extensa reforma no sistema de saúde, com a implementação do sistema de convênios odontológicos e implantação do serviço público odontológico no âmbito do Sistema Único de Saúde (SUS) 21,22. De modo compatível com esta observação, vários estudos 31,32 sublinham a importância do cirurgião-dentista na redução da carga da doença, através de sua participação nos esforços preventivos, antecipação do diagnóstico, orientação dos tratamentos e reabilitação dos pacientes.
Ao sintetizar indicações de literatura internacional sobre utilização de serviços de saúde, com menção especial aos recursos de diagnóstico precoce do câncer, Lorant et al. 33 apontaram a vacinação de idosos contra a gripe como uma das campanhas com menor diferencial de utilização entre os estratos sociais. Realizada anualmente na cidade de São Paulo desde 1996, a vacinação contra a gripe para pessoas de 60 anos ou mais tem atingido cobertura ponderável; e a incorporação do exame bucal nesse programa tem sido crescente desde 2001. Esta observação visa sublinhar a importância da participação do cirurgião-dentista nessas campanhas.

Ao reunir pessoas do grupo etário de maior risco para o câncer de boca e orofaringe, a vacinação contra a gripe pode constituir importante implemento para os esforços de diagnóstico precoce de lesões suspeitas ou pré-cancerígenas na boca e orofaringe e para a difusão de orientações acerca do auto-exame, em grupos de população menos susceptíveis a outras iniciativas de promoção da saúde. Não se pode, entretanto, negligenciar o risco da doença em pessoas com menos de 60 anos de idade, as quais não participam das campanhas de vacinação contra a gripe, e que deveriam ser objeto de iniciativas análogas. No que diz respeito à epidemiologia do câncer, nunca é demasiado enfatizar o valor de intervenções que podem configurar uma das raras fontes de expectativa para a reversão da tendência global de incremento da mortalidade por câncer de boca e orofaringe em nosso meio.

\section{Conclusão}

Observou-se tendência global de incremento na mortalidade (coeficientes ajustados por sexo e grupo etário) por câncer de boca e orofaringe na cidade de São Paulo, sendo que o câncer de língua foi responsável por mais de um terço desses óbitos. A observação de elevada magnitude e tendência crescente para a mortalidade por câncer de localizações não especificadas da boca e orofaringe sugere necessidade de implementar medidas visando a antecipação do diagnóstico e a introdução precoce dos recursos terapêuticos disponíveis. 


\section{Resumo}

Objetivou-se descrever e explorar analiticamente tendências de mortalidade por câncer de boca e orofaringe na cidade de São Paulo, Brasil, de 1980 a 2002. Dados sobre os óbitos com esta causa básica foram levantados junto à Fundação Sistema Estadual de Análise de Dados, discriminados por sexo, idade e localização anatômica. A estimação e ajuste pelo método direto dos coeficientes usaram dados de população fornecidos pelos censos de 1980, 1991 e 2000, e pela contagem populacional de 1996, realizados pelo Instituto Brasileiro de Geografia e Estatística. Foi observada tendência crescente da mortalidade devida ao agravo, a uma taxa anual de 0,72\%. Responsável por mais de um terço desses óbitos, o câncer de língua foi a categoria com mortalidade mais elevada. Câncer de lábio, gengiva e área retromolar apresentaram tendência decrescente, enquanto orofaringe e partes não especificadas da boca e orofaringe sofreram incremento de mortalidade. O monitoramento da magnitude e tendências da mortalidade por câncer pode configurar importante implemento para o planejamento de iniciativas voltadas à redução da carga de doença em nosso meio.

Neoplasias Bucais; Mortalidade; Modelos Estatísticos

\section{Colaboradores}

M. G. H. Biazevic concebeu e planejou o estudo, levantou os dados, analisou e interpretou os resultados, redigiu e revisou o texto. R. A. Castellanos orientou o trabalho e contribuiu no planejamento do estudo, na análise e interpretação dos resultados. J. L. F. Antunes apoiou a análise estatística dos resultados e colaborou no planejamento do estudo e na redação final. E. Michel-Crosato colaborou na redação e discussão dos resultados.

\section{Referências}

1. Stewart BW, Kleihues P, editors. World cancer report. Lyon: IARC Press; 2003.

2. Mahboubi E, Sayed GM. Oral cavity and pharynx. In: Schottenfeld D, Fraumeni Jr. JF, editors. Cancer epidemiology and prevention. 2nd Ed. New York: Oxford University Press; 1996. p. 583-95.

3. Hamada GS, Bos AJ, Kasuga H, Hirayama T. Comparative epidemiology of oral cancer in Brazil and India. Tokai J Exp Clin Med 1991; 16:63-72.

4. Franceschi S, Bidoli S, Herrero R, Muñoz N. Comparison of cancers of the oral cavity and pharynx worldwide: etiological clues. Oral Oncol 2000; 36:106-15.

5. Wünsch Filho V. The epidemiology of oral and pharynx cancer in Brazil. Oral Oncol 2002; 38:737-46.

6. Organização Mundial da Saúde. Classificação estatística internacional de doenças e problemas relacionados à saúde, 10a revisão. v. 1. São Paulo: Centro Colaborador da OMS para a Classificação de Doenças em Português; 1995.

7. Mirra AP, Latorre MRDO, Veneziano DB, organizadores. Aspectos epidemiológicos do câncer no município de São Paulo: fatores de risco. São Paulo: Registro de Câncer de São Paulo; 2003.

8. Organização Mundial da Saúde. Manual da classificação estatística internacional de doenças, lesões e causas de óbitos - nona conferência de revisão. São Paulo: Centro Brasileiro de Classificação de Doenças em Português; 1985.

9. Laurenti R, Mello-Jorge MHP, Gotlieb SLD. A confiabilidade dos dados de mortalidade e morbidade por doenças crônicas não-transmissíveis. Ciênc Saúde Coletiva 2004; 9:909-20.

10. Instituto Brasileiro de Geografia e Estatística. Contagem da população 1996. Rio de Janeiro: Instituto Brasileiro de Geografia e Estatística; 1997.

11. Instituto Brasileiro de Geografia e Estatística. Censo demográfico, São Paulo - 1980. v. 1. Rio de Janeiro: Instituto Brasileiro de Geografia e Estatística; 1981.

12. Instituto Brasileiro de Geografia e Estatística. Censo demográfico, São Paulo - 1991. Rio de Janeiro: Instituto Brasileiro de Geografia e Estatística; 1994.

13. Instituto Brasileiro de Geografia e Estatística. Censo demográfico 2000. Características da população e dos domicílios: resultados do universo. Rio de Janeiro: Instituto Brasileiro de Geografia e Estatística; 2001.

14. Laurenti R, Mello-Jorge MHP, Lebrão ML, Gotlieb SLD. Estatísticas de saúde. São Paulo: EPU; 1987.

15. Antunes JLF. “Grow and multiply”: social development, birth rates and demographic transition in the Municipality of São Paulo, Brazil, time-series for 1900-94. Rev Bras Epidemiol 1998; 1:61-78.

16. Carvalho JAM, Garcia RA. O envelhecimento da população brasileira: um enfoque demográfico. Cad Saúde Pública 2003; 19:725-33.

17. Gaynor PE, Kilkpatrick RC. Introduction to timeseries modeling and forecasting in business and economics. New York: McGraw-Hill; 1994.

18. Antunes JLF, Waldman EA. Trends and spatial distribution of deaths of children aged 12-60 months in São Paulo, Brazil, 1980-98. Bull World Health Organ 2002; 80:391-8. 
19. Paes NA, Albuquerque MEE. Avaliação da qualidade dos dados populacionais e cobertura dos registros de óbitos para as regiões brasileiras. Rev Saúde Pública 1999; 33:33-43.

20. Vasconcelos AMN. A qualidade das estatísticas de óbito no Brasil. Revista Brasileira de Estudos Populacionais 1998; 15:115-24.

21. Antunes JLF, Biazevic MGH, Araújo ME, Tomita NE, Chinellato LEM, Narvai PC. Trends and spatial distribution of oral cancer mortality in São Paulo, Brazil, 1980-1998. Oral Oncol 2001; 37:34550.

22. Biazevic MGH. Tendências e diferenciais socioeconômicos da mortalidade por câncer bucal e de glândulas salivares no Município de São Paulo, de 1980 a 2000 [Tese de Doutorado]. São Paulo: Faculdade de Saúde Pública, Universidade de São Paulo; 2003.

23. Instituto Nacional de Câncer. Atlas de mortalidade por câncer no Brasil 1979-1999. Rio de Janeiro: Instituto Nacional de Câncer; 2002.

24. Carlini-Marlatt B. "A população é jovem e o país é quente": estimativas do consumo de álcool e tabaco no Brasil pelos dados das indústrias produtoras. Jornal Brasileiro de Dependências Químicas $2001 ; 2: 3-8$.

25. Shafey O, Cokkinides V, Cavalcante TM, Teixeira M, Vianna C, Thun M. Case studies in international tobacco surveillance: cigarette smuggling in Brazil. Tob Control 2002; 11:215-9.
26. Schlecht NF, Franco EL, Pintos J, Negassa A, Kowalski LP, Oliveira BV, et al. Interaction between tobacco and alcohol consumption and the risk of cancers of the upper aero-digestive tract in Brazil. Am J Epidemiol 1999; 150:1129-37.

27. Chaieb JA, Castellarin C. Associação tabagismoalcoolismo: introdução às grandes dependências humanas. Rev Saúde Pública 1998; 32:246-54.

28. Lima MS, Dunn J, Novo IP, Tomasi E, Reisser AA. Gender differences in the use of alcohol and psychotropics in a Brazilian population. Subst Use Misuse 2003; 38:51-65.

29. Kowalski LP. Carcinoma de boca: epidemiologia, diagnóstico e tratamento. Acta AWHO 1991; 10: 128-34.

30. Vanderlei FAB, Pereira FC, Hojaij FC, Nishio S. Importância do exame da cavidade oral no diagnóstico precoce dos tumores de boca. Rev Med (São Paulo) 1998; 77:101-12.

31. Garrafa V, Rosa LN. O dentista e o câncer de gengiva. Rev Assoc Paul Cir Dent 1971; 25:5-9.

32. Winn DM, Sandberg AL, Horowitz AM, Diehl SR, Gutkind S, Kleinman DV. Reducing the burden of oral and pharyngeal cancers. J Calif Dent Assoc 1998; 26:445-51.

33. Lorant V, Boland B, Humblet P, Deliege D. Equity in prevention and health care. J Epidemiol Community Health 2002; 56:510-6.

Recebido em 03/Jan/2005

Versão final reapresentada em 29/Dez/2005 Aprovado em 21/Fev/2006 